\title{
Reporte del Primer Consenso Colombiano de Citometría de Flujo para el estudio de trastornos hematológicos
}

\author{
Carlos Saavedra ${ }^{1}$, Sandra Milena Quijano², Martha Romero'1, Roberto Jaramillo³ ${ }^{3}$ Rocío Orduz ${ }^{4}$, \\ Carolina Echeverri ${ }^{5}$, Mónica Londoño ${ }^{6}$, Alberto Orfao ${ }^{7}$ \\ 1 Departamento de Patología y Laboratorios, Hospital Universitario Fundación Santa Fe de Bogotá, Bogotá, D.C., \\ Colombia \\ 2 Departamento de Microbiología, Pontificia Universidad Javeriana, Bogotá, D.C., Colombia \\ 3 Fundación Valle de Lili, Cali, Colombia \\ ${ }^{4}$ Clínica Colsanitas, S. A., Bogotá, D.C., Colombia \\ 5 Hospital Pablo Tobón Uribe, Medellín, Colombia \\ 6 Instituto Nacional de Cancerología, Bogotá, D.C., Colombia \\ 7 Centro de Investigación del Cáncer (IBMCC-CSIC/USAL), Universidad de Salamanca, Salamanca, España
}

Los días 7 y 8 de julio de 2008 se celebró en Cali el Primer Consenso Colombiano de Citometría de Flujo para el estudio de enfermedades hematológicas, en conjunto con miembros de la comunidad científica de Colombia, Argentina, Chile, Perú y México, que incluyó médicos y profesionales del área de la salud vinculados a diferentes instituciones en las que se emplea la citometría de flujo como herramienta diagnóstica en hematología y oncología.

En este consenso colombiano, se discutieron las indicaciones médicas recomendadas para distintas neoplasias y los aspectos técnicos del procesamiento de diferentes tipos de muestras, y se evaluaron paneles inmunofenotípicos diagnósticos y aspectos instrumentales relacionados con la calibración y compensación de equipos y adquisición de datos, su análisis e interpretación, junto al informe de resultados.

Palabras clave: consenso colombiano, citometría de flujo, neoplasias hematológicas, inmunofenotipo.

Report on the First Colombian Consensus Conference for Flow Cytometric Immunophenotyping of hematological disorders

During July $7^{\text {th }}$ and $8^{\text {th }}$ of 2008, the First Colombian Consensus Conference for the Immunophenotyping of Hematological Malignancies was carried out in Cali, Colombia, with representatives from Colombia, Argentina, Chile, Perú and México. The consensus included professionals from the laboratory field and clinicians from different institutions that routinely operate clinical flow cytometry with an emphasis on lymphoma and leukemia immunophenotyping.

In this consensus medical indications for flow cytometry use in the detection of hematological malignancies, technical considerations for staining and samples preparation protocols, evaluation of diagnostic immunophenotyping panels, data acquisition and interpretation strategies were analyzed, and, finally, how to report the results.

Key words: Colombian consensus, flow cytometry, hematological malignances, immunophenotype.

Los días 7 y 8 de julio de 2008 se celebró una reunión de los miembros de la comunidad científica colombiana vinculados a diferentes instituciones a nivel nacional en las que se emplea la citometría de flujo como herramienta diagnóstica en hematología y oncología. Participaron médicos patólogos, médicos de laboratorio clínico, inmunólogos y

\section{Correspondencia:}

Carlos Saavedra, Departamento de Patología y Laboratorios, Hospital Universitario Fundación Santa Fé de Bogotá, Calle 119 №7-75, Bogotá, D.C., Colombia

Teléfono: 603 0303, extensiones 5177 y 5176; fax: 6196317 celamsaa54@yahoo.es bacteriólogos que representaban las distintas regiones del país y los principales centros dedicados a este tema. Además, asistieron profesionales de iguales características (médicos y profesionales del área de la salud) de la comunidad latinoamericana, relacionados con este campo. Éste fue el segundo encuentro de esta naturaleza realizado en Colombia; el primero se llevó a cabo en 1999, con el objetivo de diseñar una estrategia para el estudio de las leucemias agudas y no tuvo características de consenso.

En este Primer Consenso Colombiano de Citometría de Flujo se discutieron los principales temas 
relacionados con la utilización de dicha técnica en el diagnóstico y seguimiento de pacientes con enfermedades onco-hematológicas. Se analizaron diferentes aspectos, entre los que se incluyeron:

I. las indicaciones médicas;

II. los aspectos técnicos del procesamiento de las muestras de sangre periférica, médula ósea y otras muestras no sanguíneas;

III. la evaluación general de los distintos paneles diagnósticos relacionados con entidades específicas, como la leucemia linfoblástica aguda, la leucemia mieloide aguda, las gammapatías monoclonales, los síndromes linfoproliferativos crónicos B y T, los síndromes mielodisplásicos, la hemoglobinuria paroxística nocturna, la aplasia medular y los síndromes mieloproliferativos crónicos;

IV. los aspectos instrumentales relacionados con la calibración y la compensación de equipos y adquisición de datos, su análisis e interpretación, $y$

V. el informe de resultados.

El consenso fue coordinado por Alberto Orfao, y, teniendo en cuenta el Segundo Consenso Latinoamericano, se evaluaron las recomendaciones presentadas en dicho consenso y se modificaron de acuerdo con el conocimiento y la experiencia actuales (1). Se buscó una aproximación sencilla en el estudio de las muestras con relación al contexto en Colombia, en el que tenemos que considerar cuidadosamente los costos de cada estudio, tratando siempre de dar respuesta a la demanda de los hematólogos clínicos y planeando de la mejor forma de cada panel para obtener el máximo rendimiento y calidad.

Se enfatizó la utilización de la citometría de flujo para diferenciar entre una condición normal, reactiva, regenerativa o neoplásica y, con relación a esta última, se destacó la posibilidad de detectar distintos grupos de neoplasias hematológicas en el mundo desarrollado y, también, en países como el nuestro, teniendo en cuenta que no existe un registro nacional sobre la incidencia de neoplasias hematopoyéticas en Colombia.

\section{Indicaciones médicas}

Entre las indicaciones médicas para los estudios de citometría de flujo se consideraron cuatro áreas de aplicación y seis grupos de enfermedades hematológicas, de forma similar a lo evaluado en el Segundo Consenso Latinoamericano (1) (tabla 1).
Respecto a este último consenso, se hicieron modificaciones específicas en lo que se refiere al diagnóstico de la leucemia linfoblástica aguda y la leucemia mieloide aguda, en el cual la citometría de flujo tiene un papel definido para su confirmación. Además, se incluyó como nueva indicación el estudio de los síndromes mielodisplásicos con fines de diagnóstico y clasificación fenotípica.

En este sentido, se plantearon los siguientes criterios que permiten la identificación de grupos de riesgo en los síndromes mielodisplásicos: cuantificación del porcentaje de blastos, identificación de fenotipos aberrantes en los mieloblastos, características inmunofenotípicas de otras subpoblaciones celulares (neutrófilos, monocitos, serie eritroide, etc.) y la utilidad del inmunofenotipo para el rastreo de alteraciones cromosómicas (translocaciones, deleciones, etc.). Igualmente, en el seguimiento de la efectividad del tratamiento, principalmente en la evaluación de enfermedad residual mínima para el seguimiento de la leucemia linfoblástica aguda, la leucemia mieloide aguda y otras hemopatías malignas (tabla 1).

\section{Procesamiento de las muestras}

Para el procesamiento de diversos tipos de muestras es recomendable basarse en los protocolos establecidos en diferentes consensos, teniendo en cuenta el tipo de muestra y la localización del marcador que se requiere identificar con el fin de seleccionar protocolos de marcación en la membrana o en el citoplasma, o de detección simultánea de marcadores de membrana y citoplásmicos $(1,2)$. Para las moléculas intracelulares se deben realizar pasos consecutivos de permeabilización y lisis, precedidos de procesos de fijación previos a la marcación con anticuerpos, aunque se obtenga una disminución de la afinidad entre el anticuerpo y la molécula intracelular. Por otra parte, cuando se busca identificar moléculas de superficie, habitualmente deben realizarse pasos de lisis, lavado y marcación de las muestras biológicas.

Además, según el tipo de muestra (por ejemplo, líquidos corporales, biopsias de tejido linfático y no linfático, entre otras), puede ser conveniente emplear perlas o esferas fluorescentes como control interno de calibración del equipo, en términos de intensidad media de fluorescencia, y para poder cuantificar en términos absolutos el número de células presentes en la muestra por unidad de volumen. En algunas muestras con escasa viabilidad y concentración celular (por ejemplo, 
líquido cefalorraquídeo), se recomienda reducir al máximo la manipulación y hacer énfasis en los tiempos máximos de procesamiento después de la obtención de la muestra, para preservar el mayor número de células posible y así lograr una mayor sensibilidad.

En la tabla 2 se presentan las recomendaciones sobre el manejo de muestras en términos de tiempos máximos de procesamiento, lavados, uso de estabilizantes, centrifugación, anticoagulantes y el tipo de solución para su transporte.

\section{Paneles inmunofenotípicos para el estudio de leucemia linfoblástica aguda}

Para la elaboración de los paneles inmunofenotípicos, se tuvo en consideración:

i. Ia indicación médica, es decir, cuál es la pregunta que plantea el médico para que sea resuelta, en parte o en su totalidad, con el estudio de laboratorio, la elección del tipo de muestra más adecuado para el estudio (sangre periférica, médula ósea, ganglio linfático, etc.);

ii. la evaluación de los marcadores seleccionados para resolver dicha demanda, así como los clones de anticuerpos conjugados con los fluorocromos adecuados y en qué combinaciones, y

iii. la localización del antígeno (superficie celular, intracelular, soluble, etc.) para determinar la técnica de marcación más adecuada.

En el caso de neoplasias linfáticas de precursores de células $B$ y $T$, se recomienda el estudio por inmunofenotipo con el fin de confirmar el diagnóstico de leucemia aguda y de diferenciar estas entidades (leucemia linfoblástica aguda) de neoplasias de origen mieloide o de otras enfermedades como las enfermedades linfoproliferativas (3).

Tabla 1. Indicaciones médicas para la determinación de inmunofenotipo mediante citometría de flujo.

\begin{tabular}{|c|c|c|c|c|c|c|}
\hline Utilidad & LLA & LMA & SLPC & SMD & SMP & GM \\
\hline Diagnóstico & Sí & Sí & Sí & Sí ${ }^{* a}$ & $\mathrm{No}^{\mathrm{b}}$ & Sí \\
\hline Clasificación & Sí & Sí & Sí & Sí ${ }^{*}$ & $\mathrm{No}^{\mathrm{C}}$ & No \\
\hline Pronóstico y extensión & Sí & Sí & Sí & No & No & Sí \\
\hline Seguimiento & Sí & Sí & Sí & Sí & Sí & Sí \\
\hline
\end{tabular}

LLA: leucemia linfoblástica aguda; LMA: leucemia mieloide aguda; SLPC: síndrome linfoproliferativo crónico; SMD: síndrome mielodisplásico; SMP: síndrome mieloproliferativo: GM: gammapatía monoclonal

*Información adicional por otras técnicas (morfología y citogenética)

${ }^{a}$ El inmunofenotipo puede ser utilizado para el diagnóstico diferencial entre leucemias agudas y síndromes mielodisplásicos.

${ }^{\mathrm{b}}$ Excepto en mastocitosis

c Para BCR-ABL en LMC

Tabla 2. Manejo de muestras para estudios inmunofenotípicos mediante citometría multiparamétrica.

\begin{tabular}{|c|c|c|c|c|c|c|}
\hline Tipo de muestra & Tiempo** & Lavados & Estabilizantes & Centrifugación & Anticoagulantes & $\begin{array}{l}\text { SS/PBS } \\
\text { transporte* }\end{array}$ \\
\hline Sangre periférica** * & Hasta 24 horas & Sí & No & Sí & EDTA, heparina & No \\
\hline Médula ósea & Hasta 24 horas & Sí & No & Sí & EDTA, heparina & No \\
\hline $\begin{array}{l}\text { Sangre de cordón } \\
\text { umbilical }\end{array}$ & Hasta 24 horas & No & No & No & EDTA, heparina & No \\
\hline Productos de leucoféresis & Hasta 24 horas & No & No & No & EDTA, heparina & No \\
\hline Biopsias de tejidos sólidos ${ }^{* * *}$ & 60 minutos & Sí & No & Sí & No & Sí \\
\hline $\begin{array}{l}\text { Punciones de tejidos sólidos } \\
(\text { PAAF })^{* * *}\end{array}$ & 60 minutos & Sí & No & Sí & No & Sí \\
\hline $\begin{array}{l}\text { Líquido cefalorraquídeo } \\
\text { (LCR) }\end{array}$ & $\begin{array}{l}30 \text { minutos ( } \sin \\
\text { estabilizar) }\end{array}$ & Sí & $S i^{* * * *}$ & Sí (máximo dos pasos) & EDTA & No \\
\hline Otros fluidos corporales ${ }^{* * *}$ & 60 minutos & Sí & Sí & Sí & EDTA & Sí \\
\hline
\end{tabular}

* SS (solución salina) o PBS (phosphate buffered saline) para transporte.

** Tiempo máximo comprendido entre la obtención, transporte y procesamiento de la muestra. En el caso de muestras de líquido cefalorraquídeo, si no es posible procesarlas en un tiempo inferior a 30 minutos, se recomienda el uso de estabilizantes.

*** Las muestras pueden ser estabilizadas si se requiere confirmar estudios posteriores que no pueden ser practicados durante el tiempo máximo de procesamiento.

${ }^{* * * *}$ Estudios recientes $(22,39)$ demuestran que el empleo de una solución estabilizante previene la pérdida celular debida a los efectos citotóxicos in vitro del líquido cefalorraquídeo sobre los leucocitos, manteniéndose la eficacia de la estabilización por periodos superiores a las 24-48 horas de su obtención. 
El estudio morfológico y citoquímico de los blastos debe complementarse con un análisis fenotípico y citogenético de los mismos, que demuestre de forma inequívoca que se trata de precursores linfoides $\left(\mathrm{CD}^{+} 4^{+}\right.$o $\mathrm{TdT}^{+}$o $\left.\mathrm{CD}^{2} 5^{+ \text {+débil }}\right)$ de línea $\mathrm{B}$ (CyCD79a y CD19+) o T (CD7 ${ }^{+}$y CyCD3+), según los criterios propuestos por el grupo europeo EGIL (4).

Las leucemias linfoblásticas agudas de precursores $B$ se han de subclasificar fenotípicamente, según las recomendaciones del grupo EGIL, en i) pro-B (B-I), ii) común (B-II), iii) pre-B (B-III) y iv) madura (B-IV), y las leucemias linfoblásticas T, en T-I a T-IV $(4,5)$.

Por otra parte, se han descrito distintos patrones inmunofenotípicos asociados a determinadas alteraciones genéticas presentes en los blastos leucémicos de la leucemia linfoblástica aguda de precursores $\mathrm{B}$, esenciales para poder orientar los estudios genéticos que se deben practicar. De esta manera, los adultos con leucemia linfoblástica aguda con $t(9 ; 22)$ muestran un fenotipo que se puede superponer al de un precursor B $\left(\mathrm{CD} 10^{+}\right.$, $\left.\mathrm{CD}^{+} 9^{+}, \mathrm{CyCD} 79^{+}\right)$inmaduro $\left(\mathrm{CD} 34^{+}\right.$de elevada intensidad), con expresión débil y anormalmente heterogénea de CD38; además, estas células expresan de forma aberrante CD13 débil y CD33 (6-9). Por otra parte, los blastos de leucemia linfoblástica aguda pro-B con anomalías a nivel de 11q23, frecuentemente son CD19+, CD34+, CD10-, CD20-, CD15+, CD65+ y 7.1+ (mixed-lineage leukemia, MLL). Asimismo, los casos con $\mathrm{t}(12 ; 21)$ muestran expresión de CD19+, CD10++, CD34+débil, CD20+débil, CD45-/+ y HLA-DR++. Además, las células leucémicas de los pacientes con leucemia linfoblástica aguda pre-B y $\mathrm{t}(1 ; 19)$, se caracterizan por negatividad para CD34 y CD20 $(10,11)$.

En la actualidad, se cuenta con nuevos métodos para la detección de traslocaciones cromosómicas asociadas a leucemia linfoblástica aguda de precursores B mediante citometría de flujo, empleando esferas acopladas con anticuerpos conjugados específicos de distintas proteínas de fusión (BCR-ABL, TEL-AML1, E2A-PBX y MLLAF4) con gran sensibilidad (12).

Para los estudios de seguimiento de los efectos del tratamiento de la enfermedad en situaciones de enfermedad residual mínima, además, debe tenerse presente la identificación de fenotipos aberrantes en el momento del diagnóstico (asincronismos madurativos, aumento o disminución de la expresión de antígenos, fenotipos en localización ectópica, etc.) que, en el caso de la leucemia linfoblástica aguda de precursores B, están presentes en alrededor de $98 \%$ de los casos y en las leucemias linfoblásticas agudas T, en el 100\% de los pacientes $(3,13,14)$.

En relación con la estructuración de los paneles, se recomendó lo sugerido por el consenso latinoamericano con algunas modificaciones relacionadas con marcadores de linaje y maduración $B$, subclasificación de las leucemias linfoblásticas agudas de precursores $B$, alteraciones genéticas características y marcaciones opcionales (tabla 3).

Para neoplasias de precursores $T$ se hicieron recomendaciones de características similares a las originales del consenso latinoamericano, aunque se incluye como opcional todo el panel de caracterización de células T debido a que muchos de los presentes lo utilizan en el trabajo diario de rutina, y no se le reconoce en el momento actual un valor indispensable para la clasificación de las leucemias linfoblásticas agudas T (1) con traducción en la toma de decisiones clínicas. Se recomendó una combinación de cuatro marcadores para el seguimiento de la enfermedad residual mínima, según lo señalado en la tabla 4.

\section{Paneles inmunofenotípicos para el estudio de leucemia mieloblástica aguda}

En la leucemia mieloide aguda, se consideró que el uso de la citometría de flujo confirma el diagnóstico de leucemia aguda y permite diferenciarla de otras entidades como los síndromes mielodisplásicos y los síndromes mieloproliferativos crónicos.

Respecto a los anticuerpos propuestos, se tiene en cuenta que la detección de la mieloperoxidasa en los mieloblastos es de gran utilidad para la caracterización del linaje y que, en los casos negativos para mieloperoxidasa, deben identificarse como positivos más de dos antígenos mieloides para confirmar el linaje (1). En el caso de algunas leucemias mieloides agudas con anomalías genéticas recurrentes, debe tenerse en cuenta que el porcentaje de mieloblastos puede ser inferior a $20 \%$; asimismo, se considera que en los estudios por citometría de flujo la equivalencia a la definición morfológica de mieloblasto correspondería a células precursoras que, generalmente, expresan CD34, CD117 y HLA-DR o muestran compromiso madurativo a monocito (CD34-, CD117-/+, HLADR+, CD64+fuerte y CD14-/+débil), que incluyen marcadores aberrantes detectados en el momento del diagnóstico (15). 
Tabla 3. Combinaciones de anticuerpos para la inmunofenotipificación de leucemia linfoblástica aguda de precursores B.

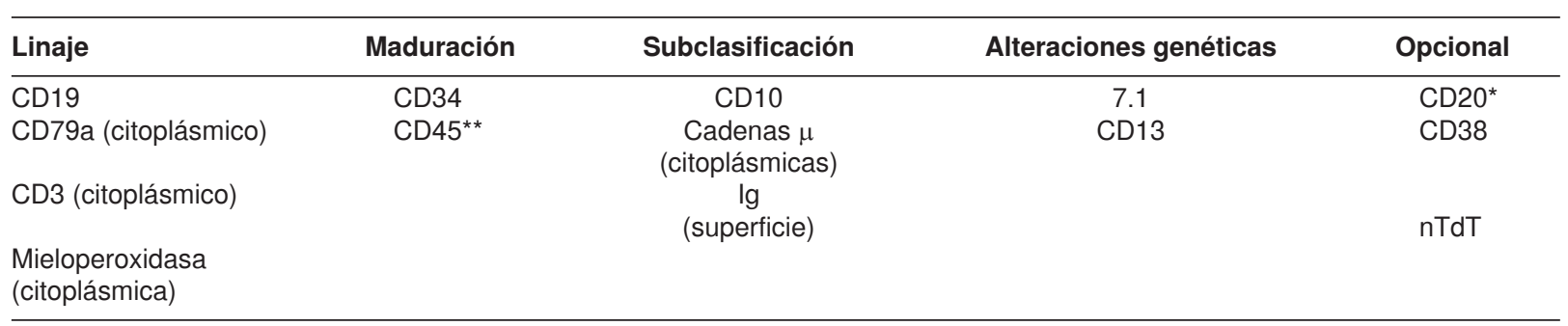

* Opcional para definir estadio madurativo

** Utilizar como marcador común si el porcentaje de infiltración es bajo.

Tabla 4. Combinaciones de anticuerpos para la inmunofenotipificación de leucemia linfoblástica aguda de precursores T.

\begin{tabular}{lccc}
\hline Linaje & Maduración & Subclasificación & Opcional \\
\hline CD3 $^{*}$ (citoplásmico) & CD34 & CD7* & CD4 \\
CD19 & CD45 & CD3* & CD8 \\
CD79a(citoplásmico) & & (superficie) & CD5 \\
MPO (citoplásmica) & & CD1a & nTdT \\
& & & CD2 \\
\hline
\end{tabular}

* Útil para estudio de enfermedad mínima residual

** Utilizar como marcador común si el porcentaje de infiltración es bajo.

MPO: mieloperoxidasa

Sin duda, la caracterización inmunológica de la leucemia promielocítica aguda por citometría de flujo permite demostrar un inmunofenotipo muy característico de la presencia de la anomalía genética característica de esta entidad -t(15;17) (reordenamiento PML- RARa)- al establecer la existencia de promielocitos aberrantes que expresan, generalmente, CD34-/+ débil, CD15-/+ débil, CD33++, CD2-/+ débil y CD13+ heterogéneo.

Otras anomalías genéticas, como la inv(16)/ $t(16 ; 16)$, se relacionan con expresión fuerte de mieloperoxidasa, CD2-/+ débil y presencia de monocitos aberrantes. Por otra parte, los casos con $t(8 ; 21)$ muestran frecuentemente expresión de CD19 y CD56, mientras que los casos con anomalías a nivel de 11q23 se asocian a fenotipo CD56+, 7.1-/+, CD2-/+ y CD19-/+ débil (16). Se recomendaron anticuerpos básicos para el diagnóstico y la clasificación de las leucemias mieloides agudas y el rastreo de alteraciones genéticas características de diferentes subtipos de la enfermedad (tabla 5).

Para efectos del seguimiento de la enfermedad residual mínima, los estudios inmunofenotípicos por citometría de flujo permiten identificar fenotipos aberrantes hasta en $85 \%$ de las leucemias mieloides agudas que en el $80 \%$ de los casos incluyen asincronismos madurativos y en $27 \%$ de casos de infidelidad de linaje y, en $20 \%$ de las leucemias mieloides agudas, aumento de la expresión antigénica (17).

\section{Paneles inmunofenotípicos para el estudio de los síndromes linfoproliferativos crónicos}

En relación con los síndromes linfoproliferativos crónicos, el uso de la citometría de flujo para el diagnóstico es de gran utilidad para:

i. establecer la posible naturaleza clonal de los linfocitos en casos de linfocitosis absoluta en adultos mayores de 40 años y sujetos con linfadenopatías superficiales;

ii. determinar el grado de extensión de la enfermedad en individuos con infiltración linfática de órganos linfáticos secundarios o de otros tejidos, y

iii.la identificación de poblaciones clonales mínimas circulantes en sangre periférica $(18,19,20)$.

Recientemente, se ha evaluado la presencia de poblaciones clonales de células $B$ en individuos sanos mayores de 40 años y se han reportado frecuencias de clones similares a los de la leucemia linfática crónica de hasta 12\% de la población (21); esto confirma la gran sensibilidad y especificidad de la citometría de flujo para el diagnóstico 
Tabla 5. Combinaciones de anticuerpos para la inmunofenotipificación de leucemia mieloblástica aguda.

\begin{tabular}{lcccc}
\hline Linajea & Maduración & Subclasificación & Alteraciones genéticas & Opcional \\
\hline MPO (citoplásmica) & HLA-DR & CD15 & CD56 & CD36 \\
CD3 (citoplásmico) & CD34 & CD13 & CD64 \\
CD19 & CD45 & CD33 & CD123 \\
CD79a (citoplásmico) & CD117a & CD11b & CD61 \\
& & & CD14 \\
\hline
\end{tabular}

a Para definir el linaje de células mieloides, debe demostrarse la expresión de dos o más marcadores asociados a linaje mieloide en los blastos según estos sean MPO+ o MPO -, respectivamente.

MPO: mieloperoxidasa

de capacidad de clonación respecto a la de la morfología convencional (18).

Asimismo, se estimaron las ventajas de la citometría de flujo en relación con los estudios histopatológicos, en el caso de punciones con aguja fina, especialmente en los linfomas $T$ y en el estudio de líquidos corporales como el líquido cefalorraquídeo, en el cual la citología tiene menor sensibilidad y especificidad respecto a la citometría de flujo (22); además, se valoró el reconocimiento en punciones con aguja fina de células patológicas de linfoma de Hodgkin (23).

En términos globales, se consideró que, sin duda, la citometría de flujo permite asignar el linaje y clasificar los síndromes linfoproliferativos crónicos T, B y NK. Los algoritmos diagnósticos propuestos se presentan de forma individualizada para los síndromes linfoproliferativos crónicos B y los síndromes linfoproliferativos crónicos T/NK en las tablas 6 y 7 .

Finalmente, se mencionó el uso de algunos anticuerpos para la evaluación del pronóstico y la importancia de la citometría de flujo en el seguimiento de la enfermedad residual mínima después del tratamiento, para lo cual deben emplearse marcaciones, al menos, de cuatro fluorescencias, con el objeto de incrementar la sensibilidad del análisis para el reconocimiento de pequeñas poblaciones de clones. Recientemente se ha demostrado, además, la gran utilidad del antígeno CD200 en la distinción entre leucemia linfática crónica (CD200++) y linfoma de células del manto (CD200-/+débil) (24).

En el caso de los síndromes linfoproliferativos crónicos $\mathrm{T}$, se recomienda realizar estudios de la capacidad de clonación por biología molecular o mediante citometría de flujo empleando anticuerpos monoclonales dirigidos a las diferentes familias TCRV $\beta$ o TCRyס $(1,25)$. De las neoplasias T, la mayoría corresponde a neoplasias $\mathrm{T}$ maduras postímicas, de las cuales, $80 \%$ son linfomas
$T$ periféricos y leucemias $T$ periféricas que, generalmente, expresan TCRa $\beta+$, mientras que las neoplasias T TCR $\gamma \delta+$ y las de células NK son aún más raras.

Entre los síndromes linfoproliferativos crónicos $\mathrm{T}$, el inmunofenotipo debe emplearse para la identificación de algunos subgrupos diagnósticos, como la leucemia prolinfocítica $\mathrm{T}$, la leucemia de linfocitos grandes granulares T/NK, la leucemia de células NK agresiva, el síndrome de Sézary y la leucemia/linfoma $\mathrm{T}$ del adulto, para lo que habitualmente se requiere la utilización de paneles inmunofenotípicos adicionales, más amplios.

Tabla 6. Combinaciones de anticuerpos para la inmunofenotipificación de síndromes linfoproliferativos crónicos B.

\begin{tabular}{lcc}
\hline Tamización & Clasificación & Opcional \\
\hline Cadenas ligeras kappa & CD5* & FMC7 \\
Cadenas ligeras lambda & CD23 & CD43 \\
CD19** & CD10 & Zap70 \\
& & (citoplásmico) \\
CD3 & CD20 & CD24 \\
CD4 & CD38 & CD22* \\
CD8 & Bc12 & CD81* \\
& & Si se sospecha TL/LZME: \\
& & CD11c \\
& & CD103 \\
& & CD25 \\
\hline * & &
\end{tabular}

* Se recomienda para estudio de enfermedad residual mínima en leucemia linfoide crónica B (1). TL: tricoleucemia; LZME: linfoma de la zona marginal esplénica

** Utilizar como marcador común

Tabla 7. Combinaciones de anticuerpos para la inmunofenotipificación de síndromes linfoproliferativos crónicos T/NK.

\begin{tabular}{lcc}
\hline Tamización & Clasificación & Opcional \\
\hline CD3 & CD7 & CD2 \\
CD4 & CD5 & Perforina (citoplásmica) \\
CD8 & CD26 & CD25 \\
CD3 & TCR $\beta \beta$ & CD30 \\
CD4 & TCR $\lambda \delta$ & CD56 \\
CD8 & Granzima (citoplásmica) & CD16 \\
& & CD52 \\
\hline
\end{tabular}


Además, el estudio inmunológico de estas neoplasias nos permite establecer correlaciones fenotípicofuncionales con la subclasificación de las células neoplásicas en células $T$ vírgenes, células $T$ de memoria o células $\mathrm{T}$ efectoras (por ejemplo, citotóxicas) (tabla 7).

Además, se revisaron estudios recientes que demuestran la utilidad de la citometría de flujo para el estudio inmunofenotípico del linfoma de Hodgkin, en el que se recomienda la evaluación de antígenos de línea B (CD19-, CD20-/+, PAX $5+$, CD79a-), antígenos relacionados con células presentadoras de antígeno (CD4+, CD36++, CD40++, CD72+, CD86++) y otros (HLA-DR++, CD30++, CD58+, CD71++, CD95++), antígenos de linaje T (CD2-, CD3-, CD5-) y antígenos de linaje mieloide (CD13-, CD15-/+, CD33-)(23).

\section{Paneles inmunofenotípicos para el estudio de gammapatías monoclonales}

En el caso de las gammapatías monoclonales, la citometría de flujo se considera útil para su diagnóstico, incluido el mieloma múltiple y su distinción de plasmocitosis reactivas. En la subclasificación de las gammapatías monoclonales, resulta útil para diferenciar entre mieloma múltiple y gammapatía monoclonal de significado indeterminado y para el seguimiento de la enfermedad residual mínima (26).

Con estos objetivos se recomendaron los siguientes anticuerpos: CD38, CD138, CD45, CD19 y el CD56 (26). Otros antígenos de utilidad reconocida incluyen CD117, CD20, CD28, CD81 y CD27, marcadores que con relativa frecuencia pueden expresarse de forma aberrante en las células plasmáticas clonales de estos pacientes. Para el estudio del mieloma múltiple es recomendable evaluar de forma simultánea la expresión de CD56, CD19, CD45 y CD38, y se ha reportado pérdida de CD19 hasta en $95 \%$ de los casos, en conjunto con disminución de la expresión de CD45 y CD38 (80\% de los casos, respectivamente) y ganancia de CD56 (50\% a $75 \%$ de los casos) $(27,28)$ (tabla 8$)$.

El diagnóstico diferencial entre mieloma múltiple y gammapatía monoclonal de significado indeterminado se realiza mediante la identificación de las células plasmáticas de médula y la evaluación, dentro de las mismas, del porcentaje de células plasmáticas patológicas/clonales, respecto al total de células plasmáticas en la muestra (26). De esta forma, si las células plasmáticas normales representan $5 \%$ o más de la población total de
Tabla 8. Combinaciones de anticuerpos para la inmunofenotipificación de gammapatías monoclonales.

\begin{tabular}{lc}
\hline $\begin{array}{l}\text { Subclasificación diagnóstica/ } \\
\text { enfermedad residual mínima }\end{array}$ & Opcional \\
\hline CD19 & $\begin{array}{c}\text { Cadenas ligeras kappa } \\
\text { (citoplasma) } \\
\text { Cadenas ligeras lambda } \\
\text { (citoplasma) }\end{array}$ \\
CD58 & \\
CD45 & \\
\hline
\end{tabular}

células plasmáticas de la médula al diagnóstico, se considera que es un rasgo característico de gammapatía monoclonal de significado indeterminado (29); por el contrario, cuando menos de $5 \%$ de las células plasmáticas muestran un fenotipo normal/policlonal, se considera que existe una elevada probabilidad de que pueda tratarse de un mieloma múltiple (29).

\section{Paneles inmunofenotípicos para el estudio de los síndromes mielodisplásicos}

El estudio de los síndromes mielodisplásicos por citometría de flujo ha demostrado ser útil para el diagnóstico de la enfermedad y su diferenciación respecto a las citopenias de otro origen 0 monocitosis reactivas, especialmente en citopenias idiopáticas de significado incierto; además, contribuye a la identificación y caracterización fenotípica de subgrupos específicos de síndromes mielodisplásicos (30). En relación con este último aspecto, resultan especialmente útiles la identificación y el recuento de blastos, y la distinción entre síndrome mielodisplásico con afectación unilineal y multilineal (30).

Los estudios inmunofenotípicos de síndromes mielodisplásicos mediante citometría de flujo han reportado diferentes alteraciones en la médula ósea, que incluyen patrones alterados de tamaño y complejidad de los mieloblastos y otras subpoblaciones celulares, aberraciones fenotípicas (aumento o disminución de la expresión antigénica, pérdida de expresión de antígenos, infidelidad de línea y bloqueos madurativos), además de alteraciones en la distribución numérica de diferentes subpoblaciones celulares $(30,31)$.

Elestudioinmunológicopermite, además, establecer un sistema de puntuación para estratificar a los pacientes en grupos de mayor o menor riesgo (32) y para el seguimiento después del tratamiento. No obstante, en este consenso, no se estimó el diseño de paneles enfocados a explorar el papel de la 
citometría de flujo en relación con el pronóstico de los síndromes mielodisplásicos, y éstos se centraron más en el diagnóstico y la clasificación de la enfermedad (tabla 1), mediante el análisis de la maduración de distintas poblaciones celulares, incluyendo neutrófilos y monocitos junto a los precursores CD34+ (tabla 9).

\section{Paneles inmunofenotípicos para el estudio de la hemoglobinuria paroxística nocturna}

Para el diagnóstico de hemoglobinuria paroxística nocturna se ha descrito que la citometría de flujo es la técnica de elección debido a su gran sensibilidad respecto a otros métodos $(33,34)$. Las recomendaciones generales incluyen el estudio de la expresión de moléculas asociadas al glicosil-fosfatidil-inositol (GPI) en, al menos, dos líneas celulares, para evaluar principalmente neutrófilos, monocitos y hematíes, en detrimento de los linfocitos y plaquetas que no se recomienda sean evaluados en la rutina diagnóstica. En publicaciones recientes, se recomienda evaluar la expresión de CD16 y CD66b o CD24, CD55 y CD59 en neutrófilos, de CD14, CD55 y CD59 en monocitos y de CD59 y CD55 en hematíes (34).

La muestraideal para el estudio de la hemoglobinuria paroxística nocturna por citometría de flujo es la sangre anticoagulada con EDTA. En principio, no se recomienda estudiar la médula ósea, con el fin de evitar problemas de interpretación de los resultados debido a la presencia en médula de formas mieloides inmaduras con expresión variable de moléculas asociadas a glicosil-fosfatidil-inositol y se analiza una muestra de sangre normal en forma paralela (34).

Se ha demostrado que la mejor combinación de marcadores fenotípicos para el estudio de la hemoglobinuria paroxística nocturna por citometría de flujo, incluye la evaluación de CD14 en monocitos y CD16 en neutrófilos (33); en el consenso se definió una combinación de cuatro marcadores para el rastreo diagnóstico de células deficitarias en glicosil-fosfatidil-inositol en el diagnóstico de la enfermedad (tabla 10), que permite identificar monocitos (CD64+ fuerte) y neutrófilos maduros (CD64- 0 +débil) en los que ha de analizarse la expresión de CD14 y CD16, respectivamente (ambos deben estar alterados).

\section{Paneles inmunofenotípicos para el estudio de} síndromes mieloproliferativos

En neoplasias mieloproliferativas, en general, la citometría de flujo ha sido de poca utilidad en
Tabla 9. Combinaciones de anticuerpos para la inmunofenotipificación de síndromes mielodisplásicos.

\begin{tabular}{ll}
\hline Marcadores fenotípicos \\
\hline CD11b/CD13/CD45/CD34 \\
CD7/CD56/CD45/CD34 \\
HLA-DR/CD117/CD45/CD34 \\
CD36/CD64/CD45/CD14 \\
TdT/MPO/CD45/CD34 \\
\hline
\end{tabular}

Tabla 10. Combinaciones de anticuerpos para la inmunofenotipificación de hemoglobinuria paroxística nocturna.

\begin{tabular}{c}
\hline Marcadores fenotípicos \\
\hline CD16/CD64/CD45/CD14
\end{tabular}

cuanto a diagnóstico, clasificación y pronóstico de este grupo de enfermedades. Por el contrario, la citometría de flujo es útil para realizar el recuento de blastos y diferenciar entre las fases crónica, acelerada y de crisis blástica, y para determinar el inmunofenotipo de los blastos en la crisis blástica (mieloide en $60 \%$ a $70 \%$ de los casos versus linfoide en $20 \%$ a $30 \%$ ) (35). No obstante, la reciente introducción de nuevas herramientas técnicas basadas en la utilización de perlas inmunomarcadas para detectar la proteína de fusión del BCR-ABL, abre las puertas, como nueva prueba muy sensible y específica, para confirmar o descartar con gran rapidez la presencia de la $t(9 ; 22)$ en casos en los que existe una sospecha diagnóstica razonada de leucemia mieloide crónica (39)

La citometría de flujo también es útil en la detección de mastocitosis sistémica, relacionada con síndromes mieloproliferativos o con neoplasia mieloide de otro tipo, como leucemia mieloide aguda o síndromes mielodisplásicos, al evaluar la expresión del marcador CD117 para la identificación de los mastocitos en su conjunto, que en las mastocitosis presentan de forma aberrante expresión de CD25 y CD2 $(36,37)$.

En el consenso se definieron algunos marcadores mieloides y linfoides útiles para el rastreo de síndromes mieloproliferativos y se propuso la detección de BCR-ABL con perlas conjugadas con anticuerpos (tabla 11).

\section{Calibración de equipos y adquisición de datos}

En el consenso se presentaron y describieron las principales características de los citómetros de flujo comercialmente disponibles en la actualidad, 
Tabla 11. Combinaciones de anticuerpos para la inmunofenotipificación del síndrome mieloproliferativo.

\begin{tabular}{c}
\hline Marcadores fenotípicos \\
\hline HLA-DR/CD117/CD45/CD34 \\
CD2/CD25/CD45/CD117 \\
Detección de BCR-ABL \\
\hline
\end{tabular}

haciendo énfasis en los nuevos instrumentos digitales y sus ventajas respecto a los equipos analógicos anteriores; asimismo, se resaltó la relevancia de emplear estrategias adecuadas de análisis de datos, como las definidas recientemente (38).

\section{Formación del personal}

En relación con el equipo humano que trabaja en el área de citometría de flujo, el grupo considera fundamental poder contar con personal con experiencia en las técnicas de marcación con anticuerpos, en el procesamiento de diversos tipos de muestras, en la adquisición del citómetro de flujo que tenga el laboratorio ,y con conocimientos de calibración y compensación del citómetro de flujo (39). También, es indispensable contar con personal que tenga experiencia y conocimientos en relación con la inmunofenotipificación de leucemias y linfomas, y que conozca los algoritmos diagnósticos. Este equipo humano debe ser multidisciplinario y ha de estar integrado con grupos de trabajo de expertos en morfología y genética, constituido por personal técnico, profesionales universitarios y médicos especialistas que mantengan una interacción activa con el médico tratante.

\section{Informe de resultados}

En el informe de los resultados, se recomienda incluir:

i) los datos generales del paciente que incluyen identificación, edad, sexo, número de historia clínica o código,

ii) características generales de la muestra (tipo de muestra, incidencias en la obtención o procesamiento, tiempo transcurrido entre la obtención y procesamiento), diagnóstico presuntivo,

iii) resultados del análisis del número de células de la muestra, que incluye valores absolutos o relativos de células patológicas y de otras poblaciones celulares identificadas,

iv) características de las poblaciones celulares relevantes (porcentaje e intensidad de expresión de antígenos), v) un comentario sobre los hallazgos del fenotipo, vi) las conclusiones del estudio realizado, y

vii)en caso de ser necesario, todas aquellas observaciones que se consideren oportunas.

Es importante incluir el panel de reactivos empleado y, de forma opcional, los gráficos más representativos del análisis (1).

\section{Conclusiones}

Durante los días de celebración del consenso colombiano, se completó con éxito la agenda propuesta en los dos días de trabajo con la presencia de representantes de las distintas instituciones y laboratorios colombianos, así como de distinguidos miembros de la comunidad latinoamericana vinculada a la citometría de flujo de Argentina, Chile, Perú y México.

En estas sesiones se discutieron ampliamente las indicaciones médicas en relación con las preguntas másfrecuentementeplanteadasenlaprácticaclínica y se utilizaron como base las recomendaciones del Segundo Consenso Latinoamericano, haciéndose algunas modificaciones en relación con lo propuesto o definido en el mismo. Estas recomendaciones incluyen paneles inmunofenotípicos de acuerdo con las actualizaciones pertinentes en cada tema, con la guía del doctor Alberto Orfao, como director del Grupo de Citometría de Flujo de la Universidad de Salamanca.

Además, se hicieron las consideraciones de costo-efectividad de los exámenes en el medio colombiano, con el fin de tratar de obtener el máximo de información posible con el mejor rendimiento y sin sacrificar la calidad de los estudios, acordándose modificaciones respecto al consenso latinoamericano en todos los tópicos, pero especialmente, en el campo de la mielodisplasia.

Por otra parte, se observa una acelerada transformación en el campo del diagnóstico fenotípico por citometría de flujo, como resultado de los avances tecnológicos asociados al desarrollo de nuevos equipos y pruebas diagnósticas, como la detección de BCR-ABL mediante citometría de flujo, para enfermedades mieloproliferativas crónicas. Finalmente, se hicieron recomendaciones para el manejo de las muestras, la adquisición y análisis de los datos, y el reporte de los resultados.

\section{Lista de participantes en el Consenso}

Alberto Orfao, Carlos Saavedra, Sandra Quijano, Carolina Echeverry, Mónica Londoño, Myriam Arévalo, Gina Cuéllar, Alba Campos, Leticia Rivera, 
Virginia Nieto, Laura Montiel, Lourdes Arriaga, Rocío Orduz, Carlos Fernández, Gema Miranda, Orlando Fals, María Ines Goldaracena, María Luisa Angarita, Ana Sánchez, Roberto Jaramillo, Martha Romero, Zulma Liliana Méndez, Carmen Lucía Roa, Jaisury Arango, Gladys Mejía, Yesenia Vidal, Rita Villa, María Teresa Urrego, María Victoria Aguirre, Patricia Hidalgo y Mauricio Gallegillos

\section{Referencias}

1. Ruiz-Arguelles A, Rivadeneyra-Espinoza L, Duque RE, Orfao A. Report on the Second Latin American Consensus Conference for flow cytometric immunophenotyping of hematological malignancies. Cytometry B. 2006;70:39-44.

2. Ruiz-Arguelles A, Duque RE, Orfao A. Report on the First Latin American Consensus Conference for Flow Cytometric Immunophenotyping of Leukemia. Cytometry. 1998;34:3942.

3. Pui $\mathbf{C H}$, Robison LL, Look AT. Acute lymphoblastic leukaemia. Lancet. 2008;371:1030-43.

4. Bene MC, Castoldi G, Knapp W, Ludwig WD, Matutes E, Orfao A, et al. Proposals for the immunological classification of acute leukemias. Leukemia. 1995;9:1783-6.

5. Lucio P, Gaipa G, van Lochem EG, van Wering ER, PorwitMacDonald A, Faria T, et al. BIOMED-I concerted action report: flow cytometric immunophenotyping of precursor B-ALL with standardized triple-stainings. BIOMED-1 concerted action investigation of minimal residual disease in acute leukemia: international standardization and clinical evaluation. Leukemia. 2001;15:1185-92.

6. Wetzler M, Dodge RK, Mrozek K, Stewart CC, Carroll AJ, Tantravahi R, et al. Additional cytogenetic abnormalities in adults with Philadelphia chromosome-positive acute lymphoblastic leukaemia: a study of the Cancer and Leukaemia Group B. Br J Haematol. 2004;124:275-88.

7. Hrusak O, Porwit-MacDonald A. Antigen expression patterns reflecting genotype of acute leukemias. Leukemia. 2002;16:1233-58.

8. Mori T, Sugita K, Suzuki T, Okazaki T, Manabe A, Hosoya $\mathbf{R}$, et al. A novel monoclonal antibody, KOR-SA3544 which reacts to Philadelphia chromosome-positive acute lymphoblastic leukemia cells with high sensitivity. Leukemia. 1995;9:1233-9

9. Tabernero MD, Bortoluci AM, Alaejos I, López-Berges MC, Rasillo A, Garcia-Sanz R, et al. Adult precursor B-ALL with $B C R / A B L$ gene rearrangements displays a unique immunophenotype based on the pattern of CD10, CD34, CD13 and CD38 expression. Leukemia. 2001;15:406-14.

10. Uckun FM, Downing JR, Gunther R, Chelstrom LM, Finnegan D, Land VJ, et al. Human $t(1 ; 19)(q 23 ; p 13)$ pre-B acute lymphoblastic leukemia in mice with severe combined immunodeficiency. Blood. 1993;81:3052-62.

11. De Zen L, Orfao A, Cazzaniga G, Masiero L, Cocito MG, Spinelli M, et al. Quantitative multiparametric immunophenotyping in acute lymphoblastic leukemia: correlation with specific genotype. I. ETV6/AML1 ALLs identification. Leukemia. 2000;14:1225-31.
12. Weerkamp F, Dekking E, Ng YY, van der Velden, V, Wai $\mathbf{H}$, Bottcher $\mathbf{S}$, et al. Flow cytometric immunobead assay for the detection of BCR-ABL fusion proteins in leukemia patients. Leukemia. 2009;23:1106-17.

13. Pui CH, Campana D, Evans WE. Childhood acute lymphoblastic leukaemia -current status and future perspectives. Lancet Oncol. 2001;2:597-607.

14. Pui CH, Relling MV, Downing JR. Acute lymphoblastic leukemia. N Engl J Med. 2004;350:1535-48.

15. Del Canizo MC, Fernández ME, López A, Vidriales B, Villaron E, Arroyo JL, et al. Immunophenotypic analysis of myelodysplastic syndromes. Haematologica. 2003;88:402-7.

16. Orfao A, Ortuno F, de Santiago M, López A, San MJ. Immunophenotyping of acute leukemias and myelodysplastic syndromes. Cytometry A. 2004;58:62-71.

17. San Miguel JF, Vidriales MB, López-Berges C, DíazMediavilla J, Gutiérrez N, Canizo C, et al. Early immunophenotypical evaluation of minimal residual disease in acute myeloid leukemia identifies different patient risk groups and may contribute to postinduction treatment stratification. Blood. 2001;98:1746-51.

18. Costa ES, Arroyo ME, Pedreira CE, García-Marcos MA, Tabernero MD, Almeida J, et al. A new automated flow cytometry data analysis approach for the diagnostic screening of neoplastic B-cell disorders in peripheral blood samples with absolute lymphocytosis. Leukemia. 2006;20:1221-30

19. Sayala HA, Rawstron AC, Hillmen P. Minimal residual disease assessment in chronic lymphocytic leukaemia. Best Pract Res Clin Haematol. 2007;20:499-512.

20. Rawstron AC, Villamor N, Ritgen M, Bottcher S, Ghia P, Zehnder JL, et al. International standardized approach for flow cytometric residual disease monitoring in chronic lymphocytic leukaemia. Leukemia. 2007;21:956-64.

21. Nieto WG, Almeida J, Romero A, Teodosio C, López A, Henriques AF, et al. Increased frequency (12\%) of circulating chronic lymphocytic leukemia-like B-cell clones in healthy subjects using a highly sensitive multicolor flow cytometry approach. Blood. 2009;114:33-7.

22. Quijano S, López A, Manuel SJ, Panizo C, Deben G, Castilla C, et al. Identification of leptomeningeal disease in aggressive B-cell non-Hodgkin's lymphoma: improved sensitivity of flow cytometry. J Clin Oncol. 2009;27:1462-1469.

23. Fromm JR, Kussick SJ, Wood BL. Identification and purification of classical Hodgkin cells from lymph nodes by flow cytometry and flow cytometric cell sorting. Am J Clin Pathol. 2006;126:764-80.

24. Palumbo GA, Parrinello N, Fargione G, Cardillo K, Chiarenza A, Berretta S, et al. CD200 expression may help in differential diagnosis between mantle cell lymphoma and B-cell chronic lymphocytic leukemia. Leuk Res. 2009;33:1212-6.

25. Lima M, Almeida J, Santos AH, dos Anjos TM, Alguero MC, Queiros ML, et al. Immunophenotypic analysis of the TCR-Vbeta repertoire in 98 persistent expansions of CD3(+)/ TCR-alpha-beta(+) large granular lymphocytes: utility in assessing clonality and insights into the pathogenesis of the disease. Am J Pathol. 2001;159:1861-8. 
26. San Miguel JF, Gutiérrez NC, Mateo G, Orfao A Conventional diagnostics in multiple myeloma. Eur J Cancer. 2006;42:1510-9.

27. Mateo G, Castellanos M, Rasillo A, Gutiérrez NC, Montalban MA, Martin ML, et al. Genetic abnormalities and patterns of antigenic expression in multiple myeloma. Clin Cancer Res. 2005;11:3661-7.

28. Rawstron AC, Orfao A, Beksac M, Bezdickova L, Brooimans RA, Bumbea H, et al. Report of the European Myeloma Network on multiparametric flow cytometry in multiple myeloma and related disorders. Haematologica. 2008;93:431-8.

29. Ocqueteau M, Orfao A, Almeida J, Blade J, González M, García-Sanz R, et al. Immunophenotypic characterization of plasma cells from monoclonal gammopathy of undetermined significance patients. Implications for the differential diagnosis between MGUS and multiple myeloma. Am $\mathrm{J}$ Pathol. 1998;152:1655-65.

30. van de Loosdrecht AA, Alhan C, Bene MC, La Porta MG, Drager AM, Feuillard J, et al. Standardization of flow cytometry in myelodysplastic syndromes: report from the first European Leukemia Net working conference on flow cytometry in myelodysplastic syndromes. Haematologica. 2009;94:1124-34.

31. Matarraz S, López A, Barrena S, Fernández C, Jensen $\mathbf{E}$, Flores $\mathbf{J}$, et al. The immunophenotype of different immature, myeloid and B-cell lineage-committed CD34+ hematopoietic cells allows discrimination between normal/ reactive and myelodysplastic syndrome precursors. Leukemia. 2008;22:1175-83.
32. Malcovati L, Nimer SD. Myelodysplastic syndromes: diagnosis and staging. Cancer Control. 2008;15(Suppl):4-13.

33. Hernández-Campo PM, Almeida J, Acevedo MJ, Sánchez ML, Alberca I, Vidriales B, et al. Detailed immunophenotypic characterization of different major and minor subsets of peripheral blood cells in patients with paroxysmal nocturnal hemoglobinuria. Transfusion. 2008;48:1403-14.

34. Hernández-Campo PM, Almeida J, Orfao A. Paroxysmal nocturnal hemoglobinuria. Med Clin (Barc). 2008;131:617-30.

35. Cervantes F, Villamor N, Esteve J, Montoto S, Rives S, Rozman C, et al. 'Lymphoid' blast crisis of chronic myeloid leukaemia is associated with distinct clinicohaematological features. Br J Haematol. 1998;100:123-8.

36. Escribano L, Díaz-Agustin B, López A, Nuñez LR, García-Montero A, Almeida J, et al. Immunophenotypic analysis of mast cells in mastocytosis: When and how to do it. Proposals of the Spanish Network on Mastocytosis (REMA). Cytometry B Clin Cytom. 2004;58:1-8.

37. Escribano L, García AC, Nuñez R, Orfao A. Flow cytometric analysis of normal and neoplastic mast cells: role in diagnosis and follow-up of mast cell disease. Immunol Allergy Clin North Am. 2006;26:535-47.

38. Perfetto SP, Chattopadhyay PK, Roederer M. Seventeencolour flow cytometry: unraveling the immune system. Nat Rev Immunol. 2004;4:648-55.

39. Kraan J, Gratama JW, Haioun C, Orfao A, Plonquet A, Porwit A, et al. Flow cytometric immunophenotyping of cerebrospinal fluid. Curr Protoc Cytom. 2008; Chapter 6: Unit 6.25. 